\title{
A unifying point of view on output feedback designs for global asymptotic stabilization
}

\author{
V. Andrieu ${ }^{a, b} \quad$ L. Praly ${ }^{c}$

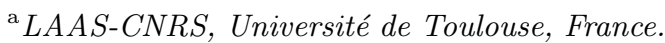 \\ ${ }^{\mathrm{b}}$ Université de Lyon 1, France, CNRS UMR 5007 LAGEP. \\ vincent. andrieu@gmail.com \\ ${ }^{\mathrm{c}}$ École des Mines de Paris \\ CAS, 35, rue Saint-Honoré, 77305 Fontainebleau Cedex \\ France. \\ praly@cas.ensmp.fr
}

\begin{abstract}
The design of output feedback for ensuring global asymptotic stability is a difficult task which has attracted the attention of many researchers with very different approaches. We propose a unifying point of view aiming at covering most of these contributions.

We start with a necessary condition on the structure of the Lyapunov functions for the closed loop system. This motivates the distinction of two classes of designs :

- the direct approach, also called control error model analysis, in which the attention is focused on directly estimating a stabilizer, and

- the indirect approach, also called dynamic error model analysis, in which the stabilization task is fulfilled for an estimated model of the system and not directly for the system itself.

We show how most available results on this topic can be reinterpreted along these lines.
\end{abstract}

Key words: output feedback, global stabilization, non linear systems

\section{Introduction.}

\subsection{Problem statement.}

We are interested in studying the solutions which have been proposed to the following stabilization problem. Given two continuous functions $f: \mathbb{R}^{n} \times \mathbb{R} \rightarrow \mathbb{R}^{n}$ and $h: \mathbb{R}^{n} \rightarrow \mathbb{R}$, find an integer $q$ and continuous functions $\nu: \mathbb{R}^{q} \times \mathbb{R} \rightarrow \mathbb{R}^{q}$ and $\varpi: \mathbb{R}^{q} \times \mathbb{R} \rightarrow \mathbb{R}$ such that the origin is a globally asymptotically stable equilibrium of the system :

$\dot{x}=f(x, u), y=h(x) \quad x \in \mathbb{R}^{n}, y \in \mathbb{R}$,

$\dot{w}=\nu(w, y), u=\varpi(w, y) \quad w \in \mathbb{R}^{q}, u \in \mathbb{R}$,

where $x$ is the state of a dynamical system to be controlled, $y$ is a measured output, $u$ is the control and $w$ is the state of a controller to be designed.

We restrict our attention here to the global case for two reasons :

(1) We want the domain of attraction to be a given open set which, in the coordinates of (1), is the whole space,

(2) For the sake of possibly achieving better performance, we address the non linear terms as they are, forbidding for instance the possibility of dominating them by functions with linear growth as typically done in the design of high gain output feedback addressing the semi-global case.

But the global case with non linear dynamics is difficult. It is known (see (Mazenc et al., 1994)) that sta- 
bilizability and observability are not sufficient for the existence of a global solution, as opposed, for instance, to the semi-global case (Atassi and Khalil, 1999; Shim and Teel, 2003; Teel and Praly, 1994) or the local case (Coron, 1994). For example, for the system

$$
\dot{\xi}_{1}=\xi_{2}, \quad \dot{\xi}_{2}=\xi_{2}^{3}+u, \quad y=\xi_{1}
$$

with input $u$, state $\left(\xi_{1}, \xi_{2}\right)$ and measured output $y$, the above problem has no solution. But it has a solution if we know that the state initial condition is in an arbitrary but given compact set (see end of section 2.3). Also the so called "separation principle" is not valid. This has been illustrated in (Kokotović, 1992) with the following system 11 :

$$
\dot{x}_{1}=-x_{1}+\left(u-x_{2}\right) x_{1}^{2}, \dot{x}_{2}=-x_{2}+x_{1}^{2}, y=x_{1} \text {. }
$$

A stabilizing state feedback is :

$$
\phi\left(x_{1}, x_{2}\right)=x_{2}
$$

and an observer for $x_{2}$ is :

$$
\dot{\hat{x}}_{2}=-\hat{x}_{2}+y^{2} .
$$

It guarantees exponential convergence of $\hat{x}_{2}$ toward $x_{2}$ on the time of existence of the solutions. Hence an output feedback obtained by designing separately stabilizer and observer as suggested by the "separation principle" is :

$$
\dot{w}=-w+y^{2}, \quad u=w .
$$

Unfortunately it does not solve our global stabilization problem since it can be checked that some solutions of the closed loop system escape to infinity in finite time.

To overcome these difficulties many different routes have been investigated by different authors and schools. Getting a complete view of all the literature is very difficult, because of its variety and its dispersion. In this paper we propose a framework for studying output feedback designs, in a unified way. For this we rely on the distinction, we shall motivate in Section 1.2, of two classes of designs :

(1) the direct approach, also called control error model analysis, in which the attention is focused on directly estimating a stabilizer,

(2) the indirect approach, also called dynamic error model analysis, in which the stabilization task is fulfilled for an estimated model of the system and not directly for the system itself.

Such a classification and the terminology we are using are not new. They are borrowed from the literature on

\footnotetext{
1 The static output feedback $u=-8 y^{5}$ solves our output feedback stabilization problem. This can be seen by completing the squares in the expression of the time derivative of $U$ in (18).
}

adaptive linear control (see (Ioannou and Sun, 1996)) and have been used in the non-linear context in (Pomet, 1989).

The paper does not contain any new result. It provides new ways of proving and viewing existing ones. It heavily relies on the dissertation (Andrieu, 2005). Also, it is certainly not a compilation of the existing literature.

\subsection{An illuminating detour.}

To motivate our forthcoming classification of output feedback designs, we consider a general interconnected system ${ }^{2}$

$$
\dot{\eta}_{s}=f_{s}\left(\eta_{s}, \eta_{e}\right) \quad, \quad \dot{\eta}_{e}=f_{e}\left(\eta_{s}, \eta_{e}\right)
$$

with $f_{s}$ and $f_{e}$ two continuous functions. As we shall see, writing the closed loop system (1) as system (6) leads to distinct interpretations depending on which part of the state $(x, w)$ is named $\eta_{s}$ or $\eta_{e}$.

Assume the origin is a globally asymptotically stable equilibrium for system (6). Then there exists a $C^{\infty}$ positive definite and radially unbounded function $V$ whose derivative along the solutions of the system is negative definite. It follows that $\eta_{s} \mapsto \operatorname{Argmin} V\left(\eta_{\eta_{e}}, \eta_{e}\right)$ is a set valued map with non-empty values. We have (see (Prieur and Praly, 2004)); (Pan et al., 2001, Section III)) :

Lemma 1 If there exists a selection $\eta_{s} \mapsto \psi\left(\eta_{s}\right) \in$ $\operatorname{Argmin}_{\eta_{e}} V\left(\eta_{s}, \eta_{e}\right)$ which is locally Hölder ${ }^{3}$ of order strictly larger than $\frac{1}{2}$, then the following holds:

(1) $U\left(\eta_{s}\right)=V\left(\eta_{s}, \psi\left(\eta_{s}\right)\right)$ is a $C^{1}$ control Lyapunov function (CLF) for the system :

$$
\dot{\eta}_{s}=f_{s}\left(\eta_{s}, u\right)
$$

whose derivative is made negative definite by the feedbach $u=\psi\left(\eta_{s}\right)$. Precisely,

$$
\eta_{s} \mapsto{ }^{d} U_{n o m}\left(\eta_{s}\right)=\frac{\partial U}{\partial \eta_{s}}\left(\eta_{s}\right) f_{s}\left(\eta_{s}, \psi\left(\eta_{s}\right)\right)
$$

is a negative definite function.

(2) There exists a continuous function $H$ satisfying :

$$
\begin{aligned}
V\left(\eta_{s}, \eta_{e}\right)= & U\left(\eta_{s}\right) \\
& +\left(\eta_{e}-\psi\left(\eta_{s}\right)\right)^{T} H\left(\eta_{s}, \eta_{e}\right)\left(\eta_{e}-\psi\left(\eta_{s}\right)\right) .
\end{aligned}
$$

\footnotetext{
2 Index "s" is to be thought as "stabilize" and index "e" as "estimating".

3 A function $f$ is said Hölder of order $\alpha$ if there exists a real number $k$ such that we have $\left|f\left(x_{1}\right)-f\left(x_{2}\right)\right| \leq k\left|x_{1}-x_{2}\right|^{\alpha}$, for all $\left(x_{1}, x_{2}\right)$.

4 In this case, we say that the feedback $\psi$ is associated to the CLF $U$.
} 
Hence with an extra condition - Hölder selection - global asymptotic stability of the origin of system (9) gives rise to the decomposition (8) which exhibits :

(1) a CLF for the $\eta_{s}$ sub-system associated to the stabilizing state feedback $\psi$;

(2) a quadratic term in $\eta_{e}-\psi\left(\eta_{s}\right)$ that, in the present context, it is tempting to interpret as an estimation error, with $\eta_{e}$ playing the role of an estimation of the stabilizer $\psi\left(\eta_{s}\right)$.

We have also the following decomposition for the time derivative of $V$ along (6) :

$$
\begin{aligned}
\dot{V}\left(\eta_{s}, \eta_{e}\right) & ={ }^{d} U_{n o m}\left(\eta_{s}\right) \\
& +\left(\eta_{e}-\psi\left(\eta_{s}\right)\right)^{T}\left[\mathfrak{A}\left(\eta_{s}, \eta_{e}\right) \dot{\eta}_{e}+\mathfrak{B}\left(\eta_{s}, \eta_{e}\right)\right],
\end{aligned}
$$

with the function ${ }^{d} U_{\text {nom }}$ defined in (7) and some functions $\mathfrak{A}$ and $\mathfrak{B}$. Since $\dot{V}$ is negative definite, $\dot{\eta}_{e}$ must be such that the positive part of :

$$
\left(\eta_{e}-\psi\left(\eta_{s}\right)\right)^{T}\left[\mathfrak{A}\left(\eta_{s}, \eta_{e}\right) \dot{\eta}_{e}+\mathfrak{B}\left(\eta_{s}, \eta_{e}\right)\right]
$$

is canceled or dominated by the negative definite function ${ }^{d} U_{\text {nom }}\left(\eta_{s}\right)$.

The decomposition (8) is the basis of the classification we propose for output feedback designs. Specifically,

(1) when the role of $\eta_{s}$ is played by the system state $x$ and the one of $\eta_{e}$ by the controller state $w$, i.e.

$$
\eta_{s}=x \quad, \quad \eta_{e}=w,
$$

then we have what we call a direct design, or a control error model analysis.

(2) Instead, when :

$$
\begin{aligned}
& \eta_{s}=w \quad, \quad \eta_{e}=x \\
& \eta_{s}=(w, y) \quad, \quad \eta_{e}=x(\bmod y=h(x)),
\end{aligned}
$$

then we have what we call an indirect design, or a dynamic error model analysis.

In each of these two classes, variations are possible depending on how much the stability margin (for instance quantified by ${ }^{d} U_{\text {nom }}$ ) is used in designing $\dot{\eta}_{e}$, as discussed about (9) above.

\subsection{System in normal form.}

To illustrate our presentation we shall quote known results for, but not only, systems in the so-called normal

\footnotetext{
${ }^{5} x(\bmod y=h(x))$ means that $\eta_{e}$ is made of the components of $x$ that are not directly given by the knowledge of $y=h(x)$. This notion makes full sense when $h(x)$ can be used as a coordinate, i.e. when the function $h$ is injective with $\left|\frac{\partial h}{\partial x}\right|$ never zero.
}

form,

$$
\begin{gathered}
\dot{z}=F\left(z, \xi_{1}\right), \\
\dot{\xi}_{1}=\xi_{2}, \ldots, \dot{\xi}_{n-1}=\xi_{n}, \\
\dot{\xi}_{n}=\bar{f}\left(z, \xi_{1}, \ldots, \xi_{n}\right)+g\left(\xi_{1}\right) u, \\
y=\xi_{1},
\end{gathered}
$$

for which a complete coordinate-free characterization is given in (Byrnes and Isidori, 1991, Corollary 5.7). This is one of the most general (nominal) structure for which we know how to design a globally asymptotically stabilizing output feedback and whose study has been initiated by (Kanellakopoulos et al., 1991) and (Marino and Tomei, 1991) and further developed for instance in (Andrieu and Praly, 2008; Andrieu et al, 2008; Freeman and Kokotović, 1996; Karagiannis et al., 2005; Krishnamurthy and Khorrami, 2004; Jiang et al., 2004; Marino and Tomei, 2005; Polendo and Qian, 2005; Qian and Lin, 2006) (see also the references therein).

An important point to emphasize is that, as usual with systems whose dynamic is nonlinear, the coordinates play a very significant role. By changing coordinates we may have a better view on some peculiarity of the system. For instance, by choosing arbitrarily sufficiently smooth functions $a_{1}$ to $a_{n-1}$, positive, and $b_{1}$ to $b_{n-1}$, we can find another set of coordinates $\left(z, y_{1}, \ldots, y_{n}\right)$ with which the above dynamic (10) can be rewritten as 6 :

$$
\begin{aligned}
\dot{z} & =F\left(z, y_{1}\right), \\
\dot{y}_{1} & =a_{1}\left(z, y_{1}\right) y_{2}+b_{1}\left(z, y_{1}\right), \quad y=y_{1}, \\
& \vdots \\
\dot{y}_{n-1}= & a_{n-1}\left(z, y_{1}, \ldots, y_{n-1}\right) y_{n} \\
& \quad+b_{n-1}\left(z, y_{1}, \ldots, y_{n-1}\right), \\
\dot{y}_{n}= & a_{n}\left(y_{1}\right) u+b_{n}\left(z, y_{1}, \ldots, y_{n}\right),
\end{aligned}
$$

or, in compact form, as :

$$
\dot{\chi}=A(\chi, y)+B(y) u, \quad \dot{y}=C(\chi, y),
$$

with :

$$
\chi=\left(z, y_{2}, \ldots, y_{n}\right) .
$$

For example the following system :

$$
\dot{\xi}_{1}=\xi_{2}, \quad \dot{\xi}_{2}=\xi_{2}^{2}+u, \quad y=\xi_{1}
$$

is in the normal form (10). And, with the change of co-

\footnotetext{
$\overline{6}$ The change of coordinates $\left(y_{i}=\psi_{i}\left(\xi_{1}, \ldots, \xi_{i}\right)\right)_{i=1, \ldots, n}$ is recursively defined as $\psi_{i+1}=\frac{\dot{\psi}_{i}-b_{i}}{a_{i}}$ with $\psi_{1}\left(\xi_{1}\right)=\xi_{1}$
} 
ordinates :

$$
\left(\begin{array}{l}
\xi_{1} \\
\xi_{2}
\end{array}\right) \mapsto\left(\begin{array}{l}
y_{1} \\
y_{2}
\end{array}\right)=\left(\begin{array}{c}
\xi_{1} \\
\xi_{2} \exp \left(-\xi_{1}\right)
\end{array}\right),
$$

its dynamic appears as being :

$$
\dot{y}_{1}=\exp \left(y_{1}\right) y_{2}, \quad \dot{y}_{2}=\exp \left(-y_{1}\right) u, \quad y=y_{1} \text {. }
$$

Instead of the quadratic nonlinearity present in (13), we have now simply linear terms up to multiplication by strictly positive output functions.

\section{Direct design $=$ control error model analysis.}

\subsection{The context.}

The design is approached by viewing the closed loop system (1) as system (6) with the following identification :

$$
\eta_{s}=x \quad, \quad \eta_{e}=w .
$$

Lemma 1 says (ignoring the requirement of a Hölder selection!) that, if the stabilization problem is solved, then there exist a function $\psi$ and a Lyapunov function $V$ such that we have :

$$
\begin{aligned}
& V(x, w)=U(x)+(w-\psi(x))^{T} H(x, w)(w-\psi(x)) \\
& \dot{V}(x, w)={ }^{d} U_{n o m}(x) \\
&+(w-\psi(x))^{T}[\mathfrak{A}(x, w) \dot{w}+\mathfrak{B}(x, w)] \\
&<0 \quad \forall(x, w) \neq 0
\end{aligned}
$$

where,

${ }^{d} U_{n o m}(x)=\frac{\partial U}{\partial x}(x) f(x, \varpi(\psi(x), h(x)))<0 \forall x \neq 0$

These three relations can be interpreted as follows.

(1) As mentioned above, we can view $w$ as an estimator of $\psi(x)$, whose meaning is clarified below.

(2) (16) says that, for the system :

$$
\dot{x}=f(x, u),
$$

we have a CLF $U$ to which is associated the state feedback :

$$
u=\phi(x)=\varpi(\psi(x), h(x))
$$

Embedded here is a control reparameterization :

$$
u=\varpi(v, y),
$$

with $v$ the new control. This operation allows us to go from the estimated $\psi$ to the state feedback $\phi$.
For instance, in the case where the function $\psi$ is the identity map, $w$ should be an estimation of the state $x$ itself.

(3) Finally, (15) says that $\dot{w}$ must be designed to get $\dot{V}$ negative definite. This can be done by using or not the already negative term ${ }^{d} U_{n o m}$, i.e. by exploiting or not the stability margin of the state feedback.

We call this approach direct design since $w$ is "directly" estimating $\psi(x)$, the reparameterized state feedback, which is the only information we need for the stabilization of (17). But $w$ is only an estimation, hence, when implementing the control as (see (1)) :

$$
u=\varpi(w, y)=\varpi(w, h(x)),
$$

we are introducing the control error $e=\psi(x)-w$ as a disturbance. This explains why we call also this method control error model analysis.

According to this direct approach, an output feedback design consists of the following steps :

step 1 : Design a stabilizing state feedback $\phi(x)$ for system (17)

step 2 : Do a control reparameterization of this state feedback as :

$$
\phi(x)=\varpi(\psi(x), h(x)),
$$

step 3 : Design an observer, i.e. $\dot{w}$, for the reparameterized control law $\psi$ which also guarantees the negativeness of $\dot{V}$ in (15).

We can re-interpret along these lines what is proposed for instance in (Arcak and Kokotović, 2001-TAC; Polendo and Qian, 2005; Qian and Lin, 2006) and (Andrieu and Praly, 2008, Section 2).

A peculiarity of this approach is to design first a state feedback and second an observer. To illustrate it, we come back to system (2). We have seen that an output feedback, designed by following the "separation principle", may not solve the global stabilization problem. The problem is that step 3 above has not been completed. Namely, in the design of $\dot{w}$, we ignored the effect of the error between the stabilizer and its estimation (see (Praly and Arcak, 2004; Arcak, 2005)). To take care of this effect, we can go on with a Lyapunov design. Specifically, to the stabilizing state feedback (3), we can associate the CLF :

$$
U\left(x_{1}, x_{2}\right)=x_{1}^{4}+x_{2}^{2} .
$$

So following (14), we consider :

$V\left(x_{1}, x_{2}, w\right)=U\left(x_{1}, x_{2}\right)+\left(w-x_{2}\right)^{2}=x_{1}^{4}+x_{2}^{2}+\left(w-x_{2}\right)^{2}$.

With completing the squares, (15) takes the form of the following inequality : 


$$
\begin{aligned}
\dot{V} \leq-3 x_{1}^{4}-x_{2}^{2} & -2\left(w-x_{2}\right)^{2} \\
& +\left[w-x_{2}\right]\left[4 x_{1}^{5}+2\left(\dot{w}+w-x_{1}^{2}\right)\right] .
\end{aligned}
$$

where we identify ${ }^{d} U_{\text {nom }}$ as being :

${ }^{d} U_{\text {nom }}\left(x_{1}, x_{2}\right)=-\left(3 x_{1}^{4}+x_{2}^{2}\right) \quad\left(\leq-U\left(x_{1}, x_{2}\right)\right)$.

Then $\dot{V}$ is made negative definite by choosing :

$$
\dot{w}=-w+y^{2}-2 y^{5},
$$

where we see the new term $-2 y^{5}$ when compared to (4).

Unfortunately, in general, even if step 1 gives a CLF, it may not be appropriate to be used as the $U$ part in the Lyapunov function $V$ given in equation (14). As a consequence, for getting $\dot{w}$, a direct Lyapunov design based on the Lyapunov function in (14), as above, maybe intractable (see however section 2.4). Typically other techniques such as those relying on a small gain argument are used. To see how we can proceed in this case and to motivate our next result, we come back to system (2) again. First, to get some flexibility we introduce a control reparameterization $(v, y) \mapsto u=v-a(y)$, where the function $a$ is a degree of freedom and $v$ is the new control. With (3), this implies that the term $\psi\left(x_{1}, x_{2}\right)$ to be estimated by $w$ is :

$$
\psi\left(x_{1}, x_{2}\right)=x_{2}+a\left(x_{1}\right) .
$$

On the other hand, for the CLF $U$ in (18), with the feedback $v=w=\psi\left(x_{1}, x_{2}\right)+e$, and by completing squares, we get :

$$
\dot{U}\left(x_{1}, x_{2}\right) \leq-U\left(x_{1}, x_{2}\right)+4 x_{1}^{5} e .
$$

Hence, when implementing the output feedback as $v=$ $w$, the effect of $e$, the error between the stabilizer and its estimation, is quantified by the term :

$$
x_{1}^{5} e=x_{1}^{5}\left(w-x_{2}-a\left(x_{1}\right)\right) .
$$

With inequality (20), we see that the global stabilization problem is solved provided this term is integrable when evaluated along the solutions of the closed loop system. Hence, we are led to say that $w$ is a good estimate of $\psi\left(x_{1}, x_{2}\right)=x_{2}+a\left(x_{1}\right)$ if the estimation error $e$ converges to zero in such a way that this integrability property holds. Since we have :

$$
\begin{aligned}
\overparen{w-x_{2}-a\left(x_{1}\right)}= & -\left[1+a^{\prime}\left(x_{1}\right) x_{1}^{2}\right]\left[w-x_{2}-a\left(x_{1}\right)\right] \\
& +\left[\dot{w}+w-a\left(x_{1}\right)-x_{1}^{2}+a^{\prime}\left(x_{1}\right) x_{1}\right],
\end{aligned}
$$

integrability of $x_{1}^{5} e$ is achieved by picking :

$$
a\left(x_{1}\right)=\frac{x_{1}\left|x_{1}\right|^{3}}{4}, \quad \dot{w}=-w+y^{2}-y|y|^{3} .
$$

This time, compared to (4), we have the new term $-y|y|^{3}$.

\subsection{Design via ISS or iISS domination.}

What has been done at the end of the above example can be done in general. The idea is to exploit the possibility that, maybe after a control reparameterization, we can find a state feedback making the system input-to-state stable (ISS) or integral input-to-state stable (iISS) with respect to an input disturbance (see (Andrieu et al, 2008; Freeman and Kokotović, 1993; Sontag, 1990)). This is formalized in the following statement.

Proposition 1 (ISS or iISS domination). The output feedback stabilization problem is solved if the integer $q$ and the continuous functions $\nu: \mathbb{R}^{q} \times \mathbb{R} \rightarrow \mathbb{R}^{q}$ and $\varpi: \mathbb{R}^{q} \times \mathbb{R} \rightarrow \mathbb{R}$ are such that the following holds :

(1) There exist a control reparameterization $u=$ $\varpi(v, y)$ and a corresponding state feedback $\psi$ making the system:

$$
\dot{x}=f(x, \varpi(\psi(x)+e, h(x)))
$$

$(\gamma)$-iISS (respectively ISS) withe as input i.e. there exist a $C^{1}$, positive definite and radially unbounded function $U$ and a continuous function $\gamma$, zero at zero, satisfying:

$$
\dot{U}(x) \leq{ }^{d} U_{n o m}(x)+\gamma(|e|) \quad \forall(x, e),
$$

with ${ }^{d} U_{\text {nom }}$ negative definite (respectively, and radially unbounded);

(2) The state $w$ of:

$$
\dot{w}=\nu(w, y)
$$

is an estimate of $\psi(x)$ such that $\gamma(|w-\psi(x)|)$ is integrable (respectively, bounded and converges to 0) along any solution of the closed loop system.

A straightforward application of this design via ISS domination yields the following result established in (Andrieu and Praly, 2008) for systems in the normal form (10).

\section{Proposition 2 If :}

(1) the sub-system $\dot{z}=F\left(z, y_{1}\right)$ is linear in $y_{1}$ and feedback linearizable;

(2) there exist functions $a_{1}$ to $a_{n-1}$, positive, and $b_{1}$ to $b_{n-1}$, a continuous function $y \mapsto K(y)$ and $a$ positive definite symmetric matrix $P$ satisfying, for all $(\chi, y)$ (see notation in (11) 7 .

\footnotetext{
7 Sufficient conditions for this assumption to hold can be found for instance in (Arcak and Kokotović, 2001-AUT; Krishnamurthy et al., 2002).
} 


$$
P \frac{\partial(A-K C)}{\partial \chi}(\chi, y)+\frac{\partial(A-K C)}{\partial \chi}(\chi, y)^{T} P<0 ;
$$

then we can solve the output feedback stabilization problem for system (10).

The first condition guarantees the existence of a continuous function $\phi$ such that the system (see (11)) :

$$
\dot{\chi}=A(\chi, y)+B(y) \phi(\chi+e, y), \quad \dot{y}=C(\chi, y)
$$

is ISS with $e$ as input. This has been established in (Freeman and Kokotović, 1993). The second condition guarantees that, by selecting :

$$
\begin{aligned}
\dot{w}=A\left(w+\int_{0}^{y} K(s) d s, y\right)+B(y) u & \\
& -K(y) C\left(w+\int_{0}^{y} K(s) d s, y\right),
\end{aligned}
$$

we get that $e=w+\int_{0}^{y} K(s) d s-\chi$ is bounded and converges to 0 along any solution. Hence a direct design via ISS domination (Proposition 1) can be done with the control reparameterization :

$$
u=\varpi(v, y)=\phi\left(v+\int_{0}^{y} K(s) d s, y\right)
$$

and :

$$
\psi\left(\chi, y_{1}\right)=\chi-\int_{0}^{y_{1}} K(s) d s .
$$

The first condition in Proposition 2 is not satisfactory. It remains an open problem to know if it could be replaced by the more "natural" one :

There exists a sufficiently many times differentiable function $\phi_{z}$ such that the system :

$$
\dot{z}=F\left(z, \phi_{z}(z+d)\right)
$$

is ISS, with $d$ as input.

\subsection{Design from the natural stability margin.}

It is not always possible to render a system ISS or iISS with respect to an input disturbance (see (Freeman, 1995; Chung, 1999)). In general, we only have that, for a given CLF $U$, reparameterization $\varpi$ and state feedback $\psi$, there exists a positive definite function $\rho$ such that we have :

$$
\begin{aligned}
& \frac{\partial U}{\partial x}(x) f(x, \varpi(\psi(x)+e, h(x)))<0 \\
& \forall(e, x):|e|<\rho(|x|) .
\end{aligned}
$$

In this case, the estimation of $\psi(x)$ by $w$ must be done to match the inequality $|w-\psi(x)|<\rho(x)$ along the solutions as quickly as possible and before any possible finite escape time. This estimation task is more demanding than the one involved in the ISS or iISS domination design. It has been found practicable for instance if : a. $\psi(x)$ is uniformly completely observable 8

b. a bound on the norm of $x$ can be estimated.

In this case, it is sufficient to use a "high gain observer" with a dynamical gain tuned from the bound on the norm of $x$. For instance, this bound can be obtained from a state norm estimator whose existence follows from an Input-Output to State Stability property. Precisely, we have the following result established in (Praly and Astolfi, 2005).

Proposition 3 If system (17) is stabilizable, uniformly completely observable and state norm detectabl $\xi^{9}$ then we can solve the output feedback stabilization problem.

This result is not fully satisfactory because of the assumption of state norm detectability which is too strong as compared to the one of unboundedness observability (see (Mazenc et al., 1994; Angeli and Sontag, 1999). It is an open problem to prove that the latter is sufficient.

Although in this paper we restrict our attention to global stabilization, it is useful here however to consider also the semi-global stabilization case. Indeed in this case there is no need of a state norm observer since a bound on this norm can be derived from knowing that the initial condition is in a given compact set. In this case there is even no need to vary the observer gain. For instance we have the following result established in (Teel and

8 Uniform complete observability : There exists a $C^{1}$ function $\Phi$ and an integer $m$ such that, for any solution $t \mapsto$ $\left(x(t), u_{0}(t), \ldots, u_{m-1}(t)\right)$, maximally defined on $\left(T_{-}, T_{+}\right)$, of :

$$
\dot{x}=f\left(x, u_{0}\right), \quad \dot{u}_{0}=u_{1}, \ldots, \dot{u}_{m-1}=u_{m}, y=h(x),
$$

we have, for each $t$ in $\left(T_{-}, T_{+}\right)$,

$$
\psi(x(t))=\Phi\left(y(t), y^{(1)}(t), \ldots, y^{(m)}(t), u_{0}(t), \ldots, u_{m-1}(t)\right),
$$

where $y^{(i)}$ denotes the $i$ th time derivative of the output $y$.

9 State norm detectability: There exist $C^{1}$ functions $W, \alpha$ and $\beta$, such that $\alpha$ is non-increasing in its first argument, $\beta$ is non-decreasing in its first argument and we have :

$$
\begin{aligned}
& \dot{W}(x)=\frac{\partial W}{\partial x}(x) f(x, u) \leq \alpha(W(x), u, h(x)) \quad \forall(x, u) \\
& |x| \leq \beta(W(x), h(x)) \quad \forall x, \quad \alpha(0, u, y) \geq 0 \quad \forall(u, y) .
\end{aligned}
$$

Moreover there exist a continuous function $\bar{\alpha}$, two nonnegative real numbers $c_{1}$ and $W_{*}$ and four strictly positive real numbers $c_{2}, c_{3}, \sigma$ and $\alpha_{*}$ satisfying :

$$
\begin{array}{r}
\alpha\left(\left(1+c_{3}\right) W+c_{1}, u, y\right)+c_{2} \leq\left[1+c_{3}\right] \alpha(W, u, y) \\
\alpha(W, u, y) \leq \bar{\alpha}(u, y), \quad \forall(W, u, y) \\
\alpha(W, u, y) \leq-\alpha_{*} \quad \forall(W, u, y): W \geq W_{*},|u|+|y| \leq \sigma .
\end{array}
$$


Praly, 1994) (see also (Atassi and Khalil, 1999; Shim and Teel, 2003)).

Proposition 4 If the origin is globally asymptotically stabilizable by a sufficiently smooth feedback and the system state $x$ is completely uniformly observable, then the origin is semi-globally stabilizable by dynamic output feedback.

Hence for instance, by tuning the gain $k$ and the level of saturation function sat in the output feedback :

$$
\begin{gathered}
\dot{\hat{x}}_{1}=\hat{x}_{2}+k\left(y-\hat{x}_{1}\right), \quad \dot{\hat{x}}_{2}=\hat{x}_{2}^{n}+u+k^{2}\left(y-\hat{x}_{1}\right), \\
u=-\operatorname{sat}\left(\hat{x}_{1}+\hat{x}_{2}+\hat{x}_{2}^{n}\right),
\end{gathered}
$$

we can solve semi-globally the asymptotic stabilization problem for the system :

$$
\dot{x}_{1}=x_{2}, \quad \dot{x}_{2}=x_{2}^{n}+u, \quad y=x_{1} .
$$

Note that this problem is not solvable globally for $n>2$.

\subsection{Without stability margin.}

There are many cases where the stability margin is unknown, though it exists. This is typically the case when we have only a weak CLF. To proceed in such cases a possibility is to apply in a straightforward manner what we learned in Lemma 1, i.e. to go with a Lyapunov design for $\dot{w}$. This has been done for instance for the example system (2) to obtain (19). As another illustration, consider the system (23) with $n=2$. A (weak) CLF is given by :

$$
U\left(x_{1}, x_{2}\right)=x_{1}^{2}+x_{2}^{2} \exp \left(-2 x_{1}\right) .
$$

Following (14), consider the function :

$V\left(x_{1}, x_{2}, w\right)=x_{1}^{2}+x_{2}^{2} \exp \left(-2 x_{1}\right)+\left(w-\psi\left(x_{1}, x_{2}\right)\right)^{2}$.

Its time derivative is :

$$
\begin{aligned}
& \dot{V}\left(y, x_{2}, w\right)= \\
& 2 x_{2}[y+u \exp (-2 y)]+2[w-\psi][\dot{w}-\dot{\psi}] .
\end{aligned}
$$

Our objective is to define $\dot{w}$ and the function $\psi$ to make this derivative non-positive. Clearly it is satisfied if, for instance, we have :

$$
\left(\begin{array}{c}
y+u \exp (-2 y) \\
\dot{w}-\dot{\psi}
\end{array}\right)=-M\left(y, x_{2}, w\right)\left(\begin{array}{c}
x_{2} \\
w-\psi\left(y, x_{2}\right)
\end{array}\right)
$$

where $M$ is any matrix with non-negative symmetric part. Indeed in this case, we get :

$$
\begin{aligned}
& \dot{V}\left(y, x_{2}, w\right)= \\
& \quad-\left(\begin{array}{ll}
x_{2} & w-\psi\left(y, x_{2}\right)
\end{array}\right) M\left(\begin{array}{c}
x_{2} \\
w-\psi\left(y, x_{2}\right)
\end{array}\right) \leq 0 .
\end{aligned}
$$

The difficulty is that the solution $(u, \dot{w})$ of $(24)$ cannot depend on $x_{2}$. One possible way to satisfy this constraint is to choose :

$$
\psi\left(x_{1}, x_{2}\right)=x_{1}, \quad M\left(y, x_{2}, w\right)=\left(\begin{array}{rr}
0 & -1 \\
1 & 1
\end{array}\right) .
$$

This yields the output feedback :

$$
u=(w-2 y) \exp (2 y), \quad \dot{w}=-(w-y) .
$$

It can be checked, by means of an invariance principle, that it does solve the output feedback stabilization problem.

More generally, when system (17) is affine in the control, i.e. we have :

$$
\dot{x}=f(x)+g(x) u,
$$

a direct Lyapunov design goes by considering the function :

$$
V(x, w)=U(x)+|\mathfrak{h}(w, x)-\psi(x)|^{2},
$$

where the functions $U, \mathfrak{h}$ and $\psi$ are to be defined so that $U$ is a (possibly weak) CLF for (25), with associated state feedback $\phi(x)$, and $w \mapsto \mathfrak{h}(w, x)$ is a diffeomorphism for all $x$. Here we have written the term $(w-\psi(x))^{T} H(x, w)(w-\psi(x))$ of (8) more simply as $|\mathfrak{h}(w, x)-\psi(x)|^{2}$. The derivative is :

$$
\begin{aligned}
\dot{V}(x, w)= & { }^{d} U_{\text {nom }}(x)+L_{g} U(x)[u-\phi(x)] \\
+[\mathfrak{h}(w, x)-\psi(x)]^{T} & \\
& \quad \times\left[\frac{\partial \mathfrak{h}}{\partial w}(w, x) \dot{w}+\frac{\partial \mathfrak{h}}{\partial x}(w, x) \dot{x}-\dot{\psi}(x)\right] .
\end{aligned}
$$

It is non-positive if we have :

$$
\begin{aligned}
&\left(\begin{array}{c}
u-\phi(x) \\
\frac{\partial \mathfrak{h}}{\partial w}(w, x) \dot{w}+\frac{\partial \mathfrak{h}}{\partial x}(w, x) \dot{x}-\dot{\psi}(x)
\end{array}\right) \\
&=-M(x, w)\left(\begin{array}{c}
L_{g} U(x) \\
\mathfrak{h}(w, x)-\psi(x)
\end{array}\right),
\end{aligned}
$$

where $M$ is any matrix with non-negative symmetric part. To complete the design it remains to select the dimension $q$ of $w$, the functions $\mathfrak{h}$ and $\psi$, and the matrix $M$ so that the solution $(u, \dot{w})$ of equation (26) depends only on $y=h(x)$ and $w$. In general such a selection is very difficult to make and may be even impossible if $U$ and $\phi$ are not appropriately selected. Some conditions under which it can be done are given in (Prieur and Praly, 2004). They are satisfied for instance by passive systems whose output is the derivative of the measurement $y$ (see (Ailon and Ortega, 1993; Ortega et al., 1995)). See also (Pomet et al., 1993).

Unfortunately this route has hardly been followed. We 
think that, in spite of its difficulty, it could be very fruitful as the applications mentioned above have shown already and because no observer is needed a priori.

\section{Indirect design = Dynamic error model anal- ysis.}

\subsection{The context.}

To introduce in a simple way the indirect design based on dynamic error model analysis, we assume that the output can be taken as one coordinate. This means that $x$ can be decomposed as $x=(\chi, y)$ and the dynamic is (see (11) for an illustration) :

$$
\dot{\chi}=A(\chi, y, u) \quad, \quad \dot{y}=C(\chi, y, u) .
$$

The design is approached by viewing the system (1) as system (6) with the following identification :

$$
\eta_{s}=(w, y) \quad, \quad \eta_{e}=\chi \quad(=x(\bmod y=h(x))) .
$$

Lemma 1 says (ignoring again the extra condition) that, if the stabilization problem is solved then there exists a Lyapunov function $V$ admitting the decomposition :

$$
\begin{aligned}
V((w, y), \chi) & =U(w, y) \\
+ & (\chi-\psi(w, y))^{T} H(\chi,(w, y))(\chi-\psi(w, y))
\end{aligned}
$$

Considering $\chi-\psi(w, y)$ as an estimation error leads us to interpret the equations :

$$
\dot{w}=\nu(w, y), \quad \hat{\chi}=\psi(w, y),
$$

as those of an observer of the unmeasured state components $\chi$.

Lemma 1 says also that $U$ is a CLF for the $\eta_{s}=(w, y)$ sub-system when $\chi=\psi(w, y)$. This sub-system is :

$$
\dot{w}=\nu(w, y), \quad \dot{y}=C(\chi, y, \varpi(w, y)),
$$

with therefore $\chi$ as control and $\chi=\psi(w, y)$ as a stabilizing state feedback.

To get a better grasp on the above two comments, it is informative to rewrite (28) and (29) as :

$$
\begin{aligned}
\dot{w} & =\nu(w, y), \\
\dot{y} & =C(\hat{\chi}, y, \varpi(w, y))+d_{y}(\chi, w, y), \\
\hat{\chi} & =\psi(w, y) .
\end{aligned}
$$

This shows a system with $\hat{\chi}$ as both input and output, and disturbed by :

$$
d_{y}(\chi, w, y)=C(\chi, y, \varpi(w, y))-C(\hat{\chi}, y, \varpi(w, y)) .
$$

The presence of $d_{y}$ explains why we call (29) a model with an error in its dynamic or shortly dynamic error model. As $U$ is a CLF for the undisturbed part of (30), with the input $\hat{\chi}=\psi(w, y)$ the associated stabilizing state feedback, one task in designing the controller functions $\nu, \varpi$ and indirectly $\psi$, is to achieve stabilization in spite of the presence of $d_{y}$. This has motivated many specific contributions on state feedbacks providing larger stability margin. See (Andrieu and Praly, 2008; Freeman and Kokotović, 1993; Kanellakopoulos et al., 1991; Krishnamurthy and Khorrami, 2006; Jiang et al., 2004; Lin and Qian, 2000) for instance.

On the other hand the disturbance $d_{y}$ is necessary for guaranteeing the convergence of the output $\hat{\chi}$ of (30) towards $\chi$ which is needed to transfer the stabilization property obtained for $(w, y)$ to $\chi$. It is because stabilization for $\chi$ is obtained in this indirect way that we call this design indirect.

Furthermore, we remark that, if (28) is indeed an observer of $\chi$, then the set $\{(\chi, y, w): \chi=\psi(w, y)\}$ should be invariant for the coupled system (27),(29). In other words, we should have the identity :

$$
\begin{aligned}
A(\psi(w, y), y, \varpi(w, y)) & =\frac{\partial \psi}{\partial w}(w, y) \nu(w, y) \\
+ & \frac{\partial \psi}{\partial y}(w, y) C(\psi(w, y), y, \varpi(w, y)) .
\end{aligned}
$$

But then this implies that we have (differentiate on both side the equation $\hat{\chi}=\psi(w, y))$ :

$$
\begin{aligned}
& \dot{y}=C(\hat{\chi}, y, \varpi(w, y)) \\
& \underbrace{\dot{\hat{\chi}}=A(\hat{\chi}, y, \varpi(w, y))}_{\text {Copy of the system }}+\underbrace{\frac{d_{y}(\chi, w, y),}{\partial y}(w, y) d_{y}(\chi, w, y)}_{\text {Dynamic error }} .
\end{aligned}
$$

From all these arguments, we conclude that an output feedback design according to this indirect approach consists in the following two steps :

step 1 : Design an observer for the state unmeasured part $\chi$, i.e. a correction term $\left(d_{y}, \psi\right)$ in $(31)$, with the objective that any good property (e.g. convergence) obtained for $\hat{\chi}$ is transferred to $\chi$,

step 2 : Design a control law $\varpi$ ensuring good properties for $\hat{\chi}$ in spite of the presence of the correction term.

Most of the publications on global stabilization by output feedback can be re-interpreted along these lines. In particular this is the case of (Andrieu and Praly, 2008; Arcak, 2005; ?; Krishnamurthy and Khorrami, 2004; Marino and Tomei, 1991; Jiang et al., 2004; Marino and Tomei, 2005; Praly and Arcak, 2004).

As opposed to the case of the direct approach, in the indirect approach, the observer is designed first and then the state feedback is designed for this observer. But there may be afterwards modifications of the observer to ease the state feedback design (see (Praly, 1992; Kanellakopoulos et al., 1992; Arcak, 2005). See Section 3.3. 
To illustrate how the indirect approach works, we consider again the system in Example 1. As already seen, an observer for $x_{2}$ is obtained by simply copying the system, i.e. :

$$
\dot{\hat{x}}_{2}=-\hat{x}_{2}+y^{2} .
$$

This gives an error $e_{2}=\hat{x}_{2}-x_{2}$ which is exponentially decaying along any solution. In this case the system (31) takes the form :

$$
\dot{y}=-y+\left(u-\hat{x}_{2}\right) y^{2}+d_{y}, \quad \dot{\hat{x}}_{2}=-\hat{x}_{2}+y^{2},
$$

with $d_{y}$ having the following specific form :

$$
d_{y}=y^{2} e_{2}
$$

Then, it remains to design :

$$
u=\varpi\left(\hat{x}_{2}, y\right) \text {. }
$$

Actually by choosing :

$$
\varpi\left(y, \hat{x}_{2}\right)=\hat{x}_{2}-y
$$

we get boundedness and convergence to 0 of any solution of (33) whatever the input $t \mapsto e_{2}(t)$ is as long as it is a continuous bounded function which converges to 0 as $t$ goes to $+\infty$. With the Lyapunov function $y^{2}+e_{2}^{2}$, it can be established that the stabilization problem is solved.

Compared with the nominal but unsatisfactory output feedback (4), here we have modified the expression of $\varpi$ in (5) by introducing $-y$. Recall that, by following a direct approach, the modification was not in $\varpi$ but in the estimation of $x_{2}$.

\subsection{Design via ISS or iISS domination.}

What has been done in the above example can be formalized in the following statement.

Proposition 5 (ISS or iISS domination) The output feedback stabilization problem is solved if we can find three functions $k_{l}, k_{r}$ and $\varpi$ such that :

(1) the system:

$$
\dot{\hat{x}}=f(\hat{x}, \varpi(\hat{x}, y))+k_{l}(\hat{x}, y) d
$$

is $(\gamma)$ iISS (resp. ISS) with d as input;

(2) along the solutions of:

$$
\begin{aligned}
& \dot{x}=f(x, \varpi(\hat{x}, y)), \\
& \dot{\hat{x}}=f(\hat{x}, \varpi(\hat{x}, y))+k_{l}(\hat{x}, y) k_{r}(\hat{x}, y),
\end{aligned}
$$

$\gamma\left(\left|k_{r}(\hat{x}, y)\right|\right)$ is integrable (resp. bounded) and $\hat{x}-x$ converges to 0 .
In the context of this proposition, we have $q=n$ and :

$$
\nu(w, y)=f(w, \varpi(w, y))+k_{l}(w, y) k_{r}(w, y) .
$$

For example, a straightforward application of this design via iISS domination yields the following result established in (Andrieu and Praly, 2008) for systems in the normal form (10).

\section{Proposition 6 If :}

(1) there exist functions $a_{1}$ to $a_{n-1}$, positive, and $b_{1}$ to $b_{n-1}$, a continuous function $y \mapsto K(y)$ and $a$ positive definite symmetric matrix $P$ satisfying, for all $(\chi, y)$ (see notation in (11)),

$$
\begin{array}{r}
P \frac{\partial(A-K C)}{\partial \chi}(\chi, y)+\frac{\partial(A-K C)}{\partial \chi}(\chi, y)^{T} P \\
<-\frac{\partial C}{\partial \chi}(\chi, y)^{T} \frac{\partial C}{\partial \chi}(\chi, y) ;
\end{array}
$$

(2) there exists a sufficiently many times differentiable function $\phi_{z}$ such that the system :

$$
\dot{z}=F\left(z, \phi_{z}(z)\right)+K_{z}(y) d
$$

is $(\gamma)$ iISS, with $d$ as input and $\gamma(s)=k s^{2}$, and where $K_{z}$ is the $z$-component of $K$ above,

then we can solve the output feedback stabilization problem for system (10).

The first condition guarantees that :

$$
d_{y}=C(\chi, y)-C(\hat{\chi}, y)
$$

is square integrable along any solution of the system

$$
\begin{aligned}
& \dot{\chi}=A(\chi, y)+B(y) u, \quad \dot{y}=C(\chi, y) \\
& \dot{\hat{\chi}}=A(\hat{\chi}, y)+B(y) u+K(y)[C(\chi, y)-C(\hat{\chi}, y)]
\end{aligned}
$$

and for any input $t \mapsto u(t)$. The second condition guarantees the existence of a continuous function $\phi$ such that the system :

$$
\begin{aligned}
& \dot{\chi}=A(\chi, y)+B(y) \phi(\chi, y)+K(y) d_{y}, \\
& \dot{y}=C(\chi, y)+d_{y},
\end{aligned}
$$

is $(\gamma)$ iISS, with $\gamma(s)=k s^{2}$, and with $d_{y}$ as input. This has been established in (Kanellakopoulos et al., 1991).

Proposition 6 follows the route of domination expressed as a property of $(\gamma)$ iISS, with $\gamma(s)=k s^{2}$. We provide now an illustration of domination with a property of $(\gamma)$ 
iISS, with $\gamma(s)=k s$. Consider the system in normal form 10 :

$$
\dot{z}=3 z+2 z^{3}+y, \quad \dot{y}=z+z^{3}+u .
$$

An observer for $z$ is given by :

$$
\dot{w}=-\hat{z}-2 \hat{z}^{3}+y-4 u, \hat{z}=w+4 y .
$$

It yields the disturbance

$$
d_{y}=\left[z+z^{3}\right]-\left[\hat{z}+\hat{z}^{3}\right]
$$

which is $L^{1}$ integrable along any solution 11 Then we see that, by selecting :

$$
\phi_{z}(z)=-4 z-3 z^{3}, \quad U(z)=\sqrt{1+z^{2}}-1
$$

gives :

$$
\frac{d U}{d z}(z)\left[3 z+2 z^{3}+\phi_{z}(z)+d\right] \leq-z^{2} \sqrt{1+z^{2}}+|d| .
$$

It follows from (Andrieu and Praly, 2008), that there exists a continuous function $\phi$ such that the system :

$$
\dot{z}=3 z+2 z^{3}+y+4 d_{y}, \quad \dot{y}=z+z^{3}+\phi(z, y)+d_{y}
$$

is $(\gamma)$ iISS, with $\gamma(s)=k s$, and with $d_{y}$ as input. Hence the output feedback :

$$
\dot{w}=-\hat{z}-2 \hat{z}^{3}+y-4 u, \hat{z}=w+4 y, u=\phi(\hat{z}, y)
$$

solves the stabilization problem.

\subsection{With an observer re-design}

Step 2 may be difficult to do, in particular to cope with the correction terms or to meet some requirement. In this case, it has been proposed to redesign the observer of step 1 while executing step 2. This is done for instance in (Kanellakopoulos et al., 1992) and in (Praly, 1992) for systems for which we can find coordinates so that the dynamic is linear in the unmeasured state components, i.e. when (27) takes the form :

$$
\dot{\chi}=A(u, y) \chi+B(u, y), \dot{y}=C(u, y)^{T} \chi+d(u, y) .
$$

To illustrate this approach, we come back to system (2). We have seen that step 1 is fulfilled with the observer (32). Now, for some reason, we insist on choosing the

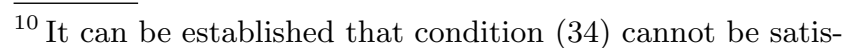
fied.

${ }^{11}$ Achieving this $L^{1}$ integrability may be difficult in general. It may be useful to modify the observer as suggested in (Arcak, 2005).
}

state feedback involved in step 2 as :

$$
u=\hat{x}_{2} .
$$

We know this leads to an unsatisfactory output feedback. So, we modify the observer in :

$$
\dot{\hat{x}}_{2}=-\hat{x}_{2}+y^{2}+m\left(\hat{x}_{2}, y\right)
$$

with the modification $m\left(\hat{x}_{2}, y\right)$ to be designed. For this, we pick 12 :

$$
V\left(y, \hat{x}_{2}, e\right)=y^{4}+\hat{x}_{2}^{2}-2 \hat{x}_{2} e_{2}+2 e_{2}^{2} .
$$

We get :

$$
\begin{aligned}
\dot{V}=-4 y^{4}+ & 2 y^{2} \hat{x}_{2}-2 \hat{x}_{2}^{2}-4 e_{2}^{2} \\
& +e_{2}\left(4 y^{5}+4 \hat{x}_{2}-2 y^{2}+2 m\left(\hat{x}_{2}, y\right)\right)
\end{aligned}
$$

which justifies the choice of the modification :

$$
m\left(\hat{x}_{2}, y\right)=-2 y^{5}-2 \hat{x}_{2}+y^{2} .
$$

\section{Domination via a dominant model.}

Up to now, both for the direct and indirect case, we have mentioned designs following a domination approach where we exploit the negativeness of ${ }^{d} U_{n o m}$, obtained for a CLF $U$ for the nominal system :

$$
\dot{x}=f(x, u) \text {. }
$$

We can push this strategy further by working only with a "dominant" approximation of this nominal system. In the linear case, the archetype of such an approach says that, by designing a high gain linear output feedback for the chain of integrators :

$\dot{x}_{1}=x_{2}, \ldots, \dot{x}_{n-1}=x_{n}, \dot{x}_{n}=u, \quad y=x_{1}$,

and by adjusting the gain, we can solve the stabilization problem by output feedback for any minimum phase linear system with relative degree $n$ (see (Khalil and Saberi, 1987) for instance). This result holds also for non linear systems if we restrict our attention to semi-global stability (see (Esfandiari and Khalil, 1992; Teel and Praly, 1995; Atassi and Khalil, 1999), see also (Praly and Jiang, 1993) for a first extension to the global case).

A way to extend it to the global case for non linear systems is to preserve the linear structure linked to a vector space but now with scalars which are no more real numbers but functions of the output. In this case, the chain of integrators is :

\footnotetext{
${ }^{12}$ We introduce the cross term $-2 \hat{x}_{2} e_{2}$ to make possible the cancellation of $\theta \frac{d}{d \hat{x}_{2}} \hat{x}_{2}^{2}$.
} 


$$
\begin{aligned}
\dot{x}_{1} & =a_{1}(y) x_{2}, \\
& \vdots \\
\dot{x}_{n-1} & =a_{n-1}(y) x_{n}, \\
\dot{x}_{n} & =a_{n}(y) u \\
y & =x_{1} .
\end{aligned}
$$

For this system, we design again a high gain linear output feedback, with linearity taken in the new sense. However, because the scalars vary along the solutions, the high gain has to be dynamical.

The chain above giving the dominant part of the model, the actual system can take the form :

$$
\begin{aligned}
\dot{x}_{1} & =a_{1}(y) x_{2}+\delta_{1}, \\
& \vdots \\
\dot{x}_{n-1} & =a_{n-1}(y) x_{n}+\delta_{n-1}, \\
\dot{x}_{n} & =a_{n}(y) u+\delta_{n}, \\
y & =x_{1},
\end{aligned}
$$

where the perturbations $\delta_{i}$ are handled via robustness. Typically, they are considered as outputs of ISS systems with the $x_{i}$ as inputs. A standard way to formalize this is to assume the following inequalities hold :

$$
\left|\delta_{i}\right| \leq \sqrt{\mu(y) V_{i}}+\mu(y) \mathfrak{b}_{i}\left(x_{1}, \ldots, x_{n}\right)
$$

with $\mu(y) \geq 0$ and where, along each solution of the system, $V_{i}$ satisfies the differential inequality :

$$
\frac{\dot{V}_{i}}{\alpha_{i}} \leq-\left(V_{i}-\mu(y) \mathfrak{b}_{i}\left(x_{1}, \ldots, x_{n}\right)^{2}\right),
$$

with $\alpha_{i}>0$. For instance, with an indirect approach, the case where the functions $\mathfrak{b}_{i}$ are :

$$
\text { - } \quad \mathfrak{b}_{i}\left(x_{1}, \ldots, x_{n}\right)=\sum_{j=1}^{i}\left|x_{j}\right|
$$

is considered in (Krishnamurthy and Khorrami, 2004);

$$
\text { - } \quad \mathfrak{b}_{i}\left(x_{1}, \ldots, x_{n}\right)=\sum_{j=i+2}^{n}\left|x_{j}\right|
$$

is considered in (Krishnamurthy and Khorrami, 2005);

- $\quad \mathfrak{b}_{i}\left(x_{1}, \ldots, x_{n}\right)=$

or

$$
\sum_{j=1}^{i}\left|x_{j}\right|^{\frac{1-d_{0}(n-i-1)}{1-d_{0}(n-j)}}+\left|x_{j}\right|^{\frac{1-d_{\infty}(n-i-1)}{1-d_{\infty}(n-j)}}
$$

$$
\sum_{j=i+2}^{n}\left|x_{j}\right|^{\frac{1-d_{0}(n-i-1)}{1-d_{0}(n-j)}}+\left|x_{j}\right|^{\frac{1-d_{\infty}(n-i-1)}{1-d_{\infty}(n-j)}}
$$

with $-1<d_{0} \leq d_{\infty}<\frac{1}{n-1}$ but all the $a_{i}$ are equal to 1 and $\mu$ is only a positive real number is considered in (Andrieu et al, 2008).

Note that this is still an open problem to unify these results, i.e, to find an output feedback controller when considering functions $\mathfrak{b}_{i}$ as in (36) and (37) with $\mu(y)$ and $a_{i}(y)$.

It is also possible to replace the chain of integrators by a chain of power integrators as in :

$$
\begin{aligned}
\dot{x}_{1} & =\operatorname{sign}(y)\left|x_{2}\right|^{p_{1}}+\delta_{1}, \\
& \vdots \\
\dot{x}_{n-1} & =\operatorname{sign}\left(x_{n}\right)\left|x_{n}\right|^{p_{n-1}}+\delta_{n-1}, \\
\dot{x}_{n} & =u+\delta_{n}, \\
y & =x_{1},
\end{aligned}
$$

where the $p_{i}$ are real numbers larger or equal to 1 . This is done for instance in (Polendo and Qian, 2005) (see also (Qian, 2005)), via a direct approach with assuming the existence of a positive real number $d$ and a positive real number $\mu$ such that we have :

where :

$$
\left|\delta_{i}\right| \leq \mu\left[\sum_{j=1}^{i}\left|x_{j}\right|^{\frac{r_{i}+d}{r_{j}}}\right]
$$

$$
r_{1}=1 \quad, \quad r_{i}+d=r_{i+1} p_{i}
$$

To illustrate this approach via a dominant model, consider the output feedback 13 (compare with (22)) :

$$
\begin{gathered}
\dot{\hat{x}}_{1}=\hat{x}_{2}+L q_{1}\left(\ell\left[y-\hat{x}_{1}\right]\right), \dot{\hat{x}}_{2}=u+L^{2} q_{2}\left(q_{1}\left(\ell\left[y-\hat{x}_{1}\right]\right),\right. \\
u=-L^{2} k \phi\left(\hat{x}_{1}, L^{-1} \hat{x}_{2}\right),
\end{gathered}
$$

where the functions $\phi, q_{1}$ and $q_{2}$ are defined as :

$$
\begin{gathered}
\phi\left(\hat{x}_{1}, \hat{x}_{2}\right)=\hat{x}_{2}+\hat{x}_{1}+\left\lceil\hat{x}_{1}\right\rfloor^{\frac{1}{1-p}} \\
+\left\lceil\hat{x}_{2}+\hat{x}_{1}+\left\lceil\hat{x}_{1}\right\rfloor^{\left.\frac{1}{1-p}\right\rfloor^{1+p},}\right. \\
q_{2}(s)=s+\lceil s\rfloor^{1+p}, \quad q_{1}(s)=s+\lceil s\rfloor^{\frac{1}{1-p}},
\end{gathered}
$$

with $p$ is in $(0,1)$. Given any $\mu$, we can tune the gains $k$, $\ell$ and $L$ to solve the global stabilization problem for any system whose dynamic can be described by :

$\dot{x}_{1}=x_{2} \quad, \quad \dot{x}_{2}=\delta+u, \quad y=x_{1}$,

with $\delta$ satisfying :

$$
|\delta| \leq \mu\left[\left|x_{1}\right|+\left|x_{2}\right|^{1+p}\right] .
$$

Actually, following (Andrieu et al, 2008), the output

\footnotetext{
${ }^{13}$ We use the notation $\lceil s\rfloor^{d}=\operatorname{sign}(s)|s|^{d}$.
} 
feedback (39) is designed for the chain of integrators :

$$
\dot{x}_{1}=x_{2} \quad, \quad \dot{x}_{2}=u \quad, \quad y=x_{1}
$$

but in such a way that this chain becomes a dominant model for systems (40) satisfying (41).

\section{Concluding remarks.}

The literature on output feedback is so rich that there is a need to clarify and compare the various contributions. To address this point, we have proposed a framework for studying, in a unified way, the proposed globally stabilizing output feedback designs. The core is a classification in direct versus indirect approach where direct means that the design is done to directly address the stabilization problem whereas indirect says that this problem is solved only because some kind of observer converges. By far, the indirect approach is the most frequently exploited in the theoretical contributions. Instead the direct approach is likely to be the most frequently used by control designers. We have also seen that, within the same class - direct or indirect - a wide variety of designs is possible depending on how much the stability margin of a state feedback or the convergence margin of an observer is exploited. In particular a full exploitation of these margins allows to develop further the approach to make it applicable, not to the given system, but only to a "dominant" approximation of it.

Also it is possible to combine direct and indirect techniques in the same design. This is done for instance in (Karagiannis et al., 2005) to deal with systems in normal form (10).

Although a lot of effort has been devoted to this output feedback stabilization problem, there are still many open problems. For instance, the need of observers is apparent, to reconstruct only a function of the state (a reparameterized version of the state feedback) or the state itself. To answer this need new observers going significantly beyond the linear paradigm have been proposed. See (Arcak and Kokotović, 2001-TAC; Andrieu et al, 2008; Krishnamurthy and Khorrami, 2006; Praly, 2003; Praly and Astolfi, 2005; Qian and Lin, 2006) for instance. However we are still limited with results like (21) or restricted to chain of integrators as dominant models, i.e. we are still far from having fully satisfactory results on observers with convergence independent of the solution behavior.

Also we have motivated the restriction of our attention here to the global asymptotic stabilization case in particular for forcing the designer to address the non linear terms as they are. It turns out that this specific point is hardly addressed by the available designs and certainly not by those going with a dominant approximation as those mentioned right above. This leads to question their interest for practice. In these regards direct designs not relying on any stability margin are very appealing since they exploit more the peculiarity of the system. Unfortunately they have received very little attention up to now.

Acknowledgments. We wish to express our deep gratitude to Alessandro Astolfi, Christophe Prieur and Ricardo Sanfelice for their invaluable help in improving the quality of this text.

\section{References}

A. Ailon, R. Ortega, An observer-based set-point controller for robot manipulators with flexible joints, Systems $\&$ Control Letters 21 (1993) 329-335

V. Andrieu, Bouclage de sortie et observateur. Thèse de doctorat de l'École des Mines de Paris, Spécialité : Mathématiques et Automatique. Décembre 2005.

V. Andrieu, L. Praly, Global asymptotic stabilization for nonminimum phase non linear systems admitting a strict normal form. IEEE Transactions on Automatic Control, Vol. 53, No. 5, June 2008.

V. Andrieu, L. Praly, A. Astolfi, Homogeneous approximation, recursive observer design and output feedback. SIAM J. Control Optim., Vol. 47, No. 4, pp. 1814-1850, 2008.

D. Angeli, E. Sontag, Forward completeness, unboundedness observability, and their Lyapunov characterizations, Systems \& Control Letters 38 (1999) 209-217

M. Arcak, Certainty equivalence output feedback design with circle criterion observers. IEEE Transactions on Automatic Control, Vol. 50, No. 6, June 2005.

M. Arcak, P. Kokotović, Observer-Based Control of Systems with Slope-Restricted Nonlinearities. IEEE Transactions on Automatic Control, Vol. 46, No. 7, July 2001.

M. Arcak, P. Kokotović, Nonlinear observers : a circle criterion design and robustness analysis. Automatica 37 (2001) 19231930 .

A. N. Atassi and H. K. Khalil, A separation principle for the stabilization of a class of nonlinear systems, IEEE Transactions on Automatic Control, Vol. 44, No. 9, September 1999.

C. Byrnes, A. Isidori, Asymptotic stabilization of minimum phase nonlinear systems. IEEE Transactions on Automatic Control, Vol. 36, No. 10, October 1991.

N. Chung, Input to state stability with respect to measurement disturbances for one dimensional systems. ESAIM : Control, Optimisation and Calculus of Variations, April 1999, Vol. 4, p. $99-121$

J.-M. Coron, On the stabilization of controllable and observable systems by output feedback law, Mathematics of Control, Signals and Systems (1994) 7, 187-216.

F. Esfandiari and H. K. Khalil, Output feedback stabilization of fully linearizable systems. International Journal of Control, 56:1007-1037, 1992.

R. Freeman, Global internal stabilizability does not imply global external stabilizability for small sensor disturbances. IEEE Transactions on Automatic Control, Vol. 40, No. 12, December 1995 
R. Freeman, P. Kokotović, Global robustness of nonlinear systems to state measurement disturbances. Proceedings of the 32nd IEEE Conference on Decision and Control, December 1993.

R. Freeman, P. Kokotović, Tracking controllers for systems linear in the unmeasured states, Automatica 32 (1996) 735-746

P. Ioannou, J. Sun, Robust adaptive control. Prentice Hall, 1996.

Z. P. Jiang, I. Mareels, D.J. Hill, J. Huang, A unifying framework for global regulation via nonlinear output feedback : from ISS to iISS, IEEE Transactions on Automatic Control, Vol. 49, No. 4, April 2004.

I. Kanellakopoulos, P. Kokotović, A. Morse. Adaptive OutputFeedback Control of a class of Nonlinear Systems. Proceedings of the 30th IEEE Conference on Decision and Control, December 1991.

I. Kanellakopoulos, M. Krstić, P. Kokotović, Interlaced controllerobserver design for adaptive nonlinear control. Proceedings of the of the 1992 American Control Conference, June 1992.

D. Karagiannis, Z-P. Jiang, R. Ortega, A. Astolfi, Outputfeedback stabilization of a class of uncertain non-minimumphase nonlinear systems. Automatica 41 (2005) 1609-1615.

H. Khalil, A. Saberi, Adaptive stabilization of a class of nonlinear systems using high-gain feedback. IEEE Transactions on Automatic Control, Vol. 32, No. 11, November 1987

P. Kokotović, The joy of feedback : nonlinear and adaptive, IEEE Control Systems Magazine, Vol. 12, No. 3, June 1992.

P. Krishnamurthy, F. Khorrami, and Z. P. Jiang, Global Output Feedback Tracking for Nonlinear Systems in Generalized Output-Feedback Canonical Form. IEEE Transactions on Automatic Control, Vol. 47, No. 5, May 2002.

P. Krishnamurthy, F. Khorrami, Dynamic high-gain scaling : state and output feedback with application to systems with ISS appended dynamics driven by all states. IEEE Transactions on Automatic Control, Vol. 49, No. 12, December 2004.

P. Krishnamurthy, F. Khorrami, Adaptive output-feedback stabilization and disturbance attenuation for feedforward systems with ISS appended dynamics. Proceedings of the 44 th IEEE Conference on Decision and Control, and the European Control Conference 2005, December 2005.

P. Krishnamurthy, F. Khorrami, On uniform solvability of parameter-dependent Lyapunov inequalities and applications to various problems. SIAM J. Control Optim. Vol. 45, No. 4, pp. 1147-1164.

W. Lin, C Qian, Adding one power integrator : a tool for global stabilization of high-order lower-triangular systems. Systems Es Control Letters 39 (2000) 339-354

R. Marino, P. Tomei, Global adaptive output-feedback control of nonlinear systems. Proceedings of the 30th IEEE Conference on Decision and Control, December 1991.

R. Marino, P. Tomei, A class of globally output feedback stabilizable nonlinear nonminimum phase systems. IEEE Transactions on Automatic Control, Vol. 50, No. 12, December 2005.

F. Mazenc, L. Praly, Global stabilization for nonlinear systems. Rapport interne CAS. January 1993

F. Mazenc, L. Praly, W. P. Dayawansa, Global stabilization by output feedback : Examples and Counter-Examples, Systems \& Control Letters 23 (1994) 119-125

R. Ortega, A. Loria, R. Kelly, L. Praly, On passivity-based output feedback global stabilization of Euler-Lagrange systems, Int. J. of Robust and Nonlinear Control, Vol. 5, 313-323, 1995.

Z. Pan, K. Ezal, A. Krener, P. Kokotović, Backstepping design with local optimality matching. IEEE Transactions on Automatic Control, Vol. 46, No. 7, July 2001
J. Polendo, C. Qian, A Generalized Framework for Global Output Feedback Stabilization of Genuinely Nonlinear Systems. Proceedings of the 44th IEEE Conference on Decision and Control, and the European Control Conference 2005, December 2005.

J.-B. Pomet, Sur la commande adaptative des systèmes non linéaires. Thèse de doctorat de l'École des Mines de Paris, Spécialité : Mathématiques et Automatique. Septembre 1989.

J.-B. Pomet, R. Hirschorn, W. Cebuhar, Dynamic output feedback regulation for a class of nonlinear systems. Mathematics of Control, Signals and Systems (1993) 6, 106124.

L. Praly, Asymptotic stabilization via output feedback for lower triangular systems with output dependent incremental rate. IEEE Transactions on Automatic Control, Vol. 48, No. 6, June 2003.

L. Praly, Lyapunov design of a dynamic output feedback for systems linear in their unmeasured state components. Proceedings of the 2nd IFAC Symposium on Nonlinear Control Systems (NOLCOS 1992). June 1992.

L. Praly, M. Arcak, A Sufficient Condition for Stability of Nonlinear Observer-Based Controllers. Systems \& Control Letters 53 (2004) 311-320

L. Praly, A. Astolfi, Global asymptotic stabilization by output feedback under a state norm detectability assumption. Proceedings of the 44th IEEE Conference on Decision and Control, December 2005

L. Praly, Z.-P. Jiang, Stabilization by output feedback for systems with ISS inverse dynamics, Systems \& Control Letters 21 (1993) 19-33.

C. Prieur, L. Praly, A tentative direct Lyapunov design of output feedbacks. Proceedings of the 6th IFAC Symposium on Nonlinear Control Systems (NOLCOS 2004). September 2004.

C. Qian, A Homogeneous Domination Approach for Global Output Feedback Stabilization of a Class of Nonlinear Systems, Proceedings of the 2005 American Control Conference Page(s),4708 - 4715 vol. 7 June 2005

C. Qian, W. Lin, Recursive observer design, homogeneous approximation, and nonsmooth output feedback stabilization of nonlinear systems, IEEE Transactions on Automatic Control, Vol. 51, No. 9, September 2006.

H. Shim, A. Teel, Asymptotic controllability and observability imply semi-global practical asymptotic stabilizability by sampled-data output feedback. Automatica 39 (2003) 441454.

E. Sontag, Further facts about input to state stabilization IEEE Transactions on Automatic Control, Vol. 35, No. 4, April 1990

A. Teel, L. Praly, Global stabilizability and observability imply semi-global stabilizability by output feedback, Systems \& Control Letters 22 (1994) 313-325

A.R. Teel, L. Praly : Tools for semi-global stabilization by partial state and output feedback. SIAM Journal on Control and Optimization, Vol. 33, No. 5, September 1995 